\title{
Exosomal microRNA-150-5p from bone marrow mesenchymal stromal cells mitigates cerebral ischemia/reperfusion injury via targeting B-cell translocation gene 2
}

shuo Yang ( Yangshuo4515@163.com )

Daqing Oilfield General Hospital

Xue Li

Daqing Oilfield General Hospital

Ting Bi

Daqing Oilfield General Hospital

Research

Keywords: Cerebral ischemia/reperfusion injury, MicroRNA-150-5p, B-cell translocation gene 2, Exosomes, Bone marrow mesenchymal stromal cells, Biological function

Posted Date: August 9th, 2021

DOI: https://doi.org/10.21203/rs.3.rs-779140/v1

License: (c) (1) This work is licensed under a Creative Commons Attribution 4.0 International License.

Read Full License 


\section{Abstract \\ Objective}

MicroRNA(miR)-150-5p has been investigated in many studies, while the role of exosomal miR-150-5p from bone marrow mesenchymal stromal cells (BMSCs) on cerebral ischemia/reperfusion (I/R) injury remains extensive exploration. This research aims to probe the protective effects of exosomal miR-150$5 p$ from BMSCs on cerebral I/R injury via regulating B-cell translocation gene 2 (BTG2).

\section{Methods}

BMSCs were cultured and transfected with miR-150-5p mimic, then exosomes from BMSCs were extracted. Middle cerebral artery occlusion (MCAO) rat model was established, and miR-150-5p and BTG2 levels in rat brain tissues were detected. Then gain and loss-function assays were conducted to probe the impact of exosomes, miR-150-5p and BTG2 on neurological function, pathological changes, apoptosis and inflammatory factors of MCAO rats. The binding relationship between miR-150-5p and BTG2 was validated.

\section{Results}

MiR-150-5p was decreased and BTG2 was augmented in MCAO rats. The exosomes from BMSCs could improve neurological function, pathological changes, apoptosis and reduce inflammatory factors in MCAO rats. Enriched miR-150-5p or decreased BTG2 could enhance the protective effects of exosomes from BMSCs on cerebral I/R injury. The elevated BTG2 reversed the impacts of enriched exosomal miR150-5p. BTG2 was targeted by miR-150-5p.

\section{Conclusion}

Exosomal miR-150-5p from BMSCs exerts protective effects on cerebral I/R injury via repressing BTG2. This study provided novel therapeutic strategies for treatment of cerebral I/R injury.

\section{Introduction}

Cerebral ischemia is defined as perturbations in blood flow to the brain that trigger long-term and complex metabolic and cellular pathology, inducing neuronal cell death and cerebral infarction [1]. Furthermore, the reperfusion after cerebral ischemia can induce brain injury, further causing cerebral edema, brain hemorrhage, and neuronal death. Such process is defined as cerebral ischemia/reperfusion $(\mathrm{I} / \mathrm{R})$ injury [2]. Intravenous thrombolysis and endovascular therapy are two major treatments for ischemic reperfusion injury [3]. However, the I/R injury still contributes to high mortality and mobility to patients 
worldwide [4]. Therefore, further investigation of the novel therapeutic strategies for cerebral I/R injury is necessary.

Bone marrow mesenchymal stromal cells (BMSCs) are termed as nonhematopoietic skeletal progenitor cells with salient importance for the hematopoietic microenvironment [5]. It has been validated that BMSCs and oxiracetam treatment can mitigate cerebral I/R injury [6]. Furthermore, it has been illustrated that BMSCs-derived exosomes may attenuate cell pyroptosis by promoting autophagic flux in cerebral I/R injury [7]. As small, single-membrane organelles that aggregate in proteins, lipids, nucleic acids, and glycoconjugates, exosomes also function as regulator of extracellular matrix and the mediator for signals and molecules [8]. Yu et al. have validated that the suppression of exosomes secretion slashes the inhibitory effects of conditioned medium of BMSCs on inflammatory factors in I/R injury [9]. Similarly, it has reported that exosomes from BMSCs effectively protect renal I/R injury and endoplasmic reticulum stress at incipient reperfusion stages [10]. Additionally, exosomal microRNAs (miRNAs) derived from BMSCs have been clarified to mitigate renal and myocardial I/R injury $[11,12]$. MiRNAs are a sequence of small noncoding RNAs that regulate gene expression [13]. Belonging to miRNA, miR-150 has been revealed to reduce vascular density of infarct border zone in middle cerebral artery occlusion (MCAO) rats and constrain the growth of brain microvascular endothelial cells [14]. In addition, Ou et al. have unearthed that miR-150-5p is depleted in I/R rats, while the enrichment of miR-150-5p can repress the cardiomyocyte apoptosis in I/R rats [15]. Furthermore, the bioinformatic website predicted there was a binding relationship between miR-150-5p and B-cell translocation gene 2 (BTG2). Suzuki et al. have implied that BTG2 deletion facilitates the proliferation of glial cells during cerebral hypo-perfusion [16]. Furthermore, it has been reported that BTG2 displays high levels in the corpus callosum of mice with chronic cerebral hypoperfusion [17]. Nevertheless, the efficacy of exosomal miR-150-5p in cerebral I/R injury development through mediating BTG2 necessitated further extensive exploration. Therefore, this study committed to probe the protective effects of exosomal miR-150-5p from BMSCs on the progression of cerebral I/R injury, thus to afford novel therapeutic strategies and a distinguished research direction for the treatment of cerebral I/R injury.

\section{Material And Methods}

\section{Ethic statement}

Animal experiments were conformed to the Guide to the Management and Use of Laboratory Animals issued by the National Institutes of Health. The protocol of animal experiments was approved by the Institutional Animal Care and Use Committee of Daqing Oilfield General Hospital.

\section{Laboratory animal}

Specific pathogen-free grade sprague dawley male rats (260-280 g) were purchased from GuangZhou Jennio Biotech Co., Ltd (Guangzhou, China) for the preparation of MCAO rat model. Female rats were characterized by high estrogen levels with neuroprotective function and periodic estrogen secretion. During the experiment, the estrogen level could not be calculate accurately, bringing some difficulties to 
the application of female animal model of cerebrovascular disease. Because of the instability of the female animal model, male rats were selected for the preparation of MCAO rat model.

\section{Isolation and culture of BMSCs as well as the extraction and identification of exosomes}

The fetal bovine serum (FBS) was ultra-centrifuged $\left(120,000 \times \mathrm{g}, 4^{\circ} \mathrm{C}, 18\right.$ hours) to remove exosomes in serum. The exosomes-removing FBS and penicillin-streptomycin were added into Dulbecco's modified Eagle's medium (DMEM), and the DMEM containing 10\% FBS and 1\% penicillin-streptomycin was prepared. The bone marrow cavity of femur and tibia of rats was washed with prepared culture medium in a sterile environment. The obtained bone marrow cells were seeded in a $25 \mathrm{~cm}^{2}$ culture dish for incubation. Then, the culture medium was replaced every 2 days and the morphology and growth of the cells were observed under a microscope. When the cell confluence reached to $80-90 \%$, the cells were detached with $0.25 \%$ trypsin-ethylene diamine tetraacetic acid solution and passaged in 1:2.

The BMSCs of passage 2 were detached, adjusted to $1.0 \times 10^{6} \mathrm{pc} / \mathrm{mL}$ and put into $1.5 \mathrm{~mL}$ eppendorf (EP) tubes. CD29-PE, CD45-PE, CD54-PE and CD90-PE rabbit-anti-rat monoclonal antibodies were added, respectively (BD phamingen company, $\mathrm{CA}, \mathrm{USA}$ ). A homeotype of negative control (NC) was established, reacted for 30 minutes and determined by a flow cytometer (Bio-Rad Laboratories, Hercules, CA, USA).

Cells were fixed at $20^{\circ} \mathrm{C}$ with $4 \%$ paraformaldehyde for 30 minutes, then stained with oil red $\mathrm{O}$ solution (Nanjing Jincheng Bioengineering Institute, Nanjing, China) at $20^{\circ} \mathrm{C}$ for 1 hour. Stained cells were observed with a microscope. Then, the cells were stained with $0.1 \%$ alizarin red $\mathrm{S}$ in distilled water $(\mathrm{pH}$ 4.2) at $20^{\circ} \mathrm{C}$ for 60 minutes. After staining, the cells were rinsed with distilled water for three times and observed..To quantify the stained cells, the integrated optical density (OD) and osteogenic effects of lipid drops were determined through Image-pro plus ((Media Cybernetics, MD, USA) [18].

When the confluence of BMSCs reached to about $80 \%$, the supernatant was removed from the culture medium, and BMSCs were cultured with 10\% exosomes-removing FBS culture medium for 48 hours. The collected supernatant was centrifuged at $500 \mathrm{~g}$ for 15 minutes, and at $2000 \mathrm{~g}$ for 15 minutes. Then, the vacuole was removed by centrifugation at $10,000 \times \mathrm{g}$ at $4^{\circ} \mathrm{C}$ for 20 minutes. Thereafter, the supernatant was filtered with a 0.22 micron filter, and then ultra-centrifuged at $110,000 \times \mathrm{g}$ at $4^{\circ} \mathrm{C}$ for 70 minutes. Afterwards, the supernatant was resuspended with $100 \mu \mathrm{L}$ sterile phosphate buffered saline for downstream experiments. All of these processes above were performed in Beckman Coulter (TL-100) with TLS-55 oscillating bucket rotor at $4^{\circ} \mathrm{C}$ [19]. Then, the exosomes were stained with $3 \%$ aqueous phosphotungstic acid for 5 minutes. Finally, the samples were observed with transmission electron microscope running at $100 \mathrm{kV}$, and the particle size of the exosomes was detected with ZetaView (Particle Metrix, Meerbusch, Germany). The marker proteins CD63, CD9 and CD81 were detected by Western blot assay.

\section{Cell transfection}


Lipofectamine 3000 (Thermo Fisher Scientific Inc., Waltham, MA, USA) was used for cell transfection. Mimic-NC and miR-150-5p-mimic plasmids were transfected into BMSCs to obtain exosomes [20].

\section{MCAO rat model establishment}

MCAO rat model was constructed with modified nylon suture method. Rats were fasted before ischemic injury. Then, rats were anaesthetized by intraperitoneal injection of $4 \%$ chloral hydrate $(0.3 \mathrm{mg} / 100 \mathrm{~g})$ and a midline ventral neck incision was conducted. The right common carotid artery (CCA), external carotid artery (ECA) and internal carotid artery (ICA) were exposed. The CCA and the ECA were ligated through the ventral midline neck incision. Ischemia was caused through blocking the middle cerebral artery (MCA) of rats with the insertion of nylon monofilament sutures into the ICA. The filaments were held for 2 hours and the monofilament was removed to restore ICA-MCA blood perfusion for around 24 hours. The rats temperature was maintained at $37^{\circ} \mathrm{C}$ by a thermostatically controlled heating pad until rats recovered from surgery.

The experimental groups were as follows: sham group (only vessels were isolated without ligation), MCAO group (MCAO rat model was established), Exo group (injection of BMSCs-exosomes), Exo + mimic$\mathrm{NC}$ group (injection of exosomes from BMSCs that transfected with mimic-NC), Exo + miR-150-5p-mimic group (injection of exosomes from BMSCs that transfected with miR-150-5p-mimic), Exo + short hairpin RNA (sh)-NC group (injection of BMSCsexosomes and that of lentivirus cloned with sh-NC sequence), Exo + sh-BTG2 group (injection of BMSCs-exosomes and that of lentivirus cloned with sh-BTG2 sequence), Exo + miR-150-5p-mimic + overexpression (oe)-NC group (injection of exosomes from BMSCs that transfected with miR-150-5p-mimic and that of lentivirus that cloned with oe-NC sequence), Exo + miR-150-5p-mimic + oe-BTG2 group (injection of exosomes from BMSCs that transfected with miR-150$5 p$-mimic and that of lentivirus cloned with oe-BTG2 sequence) [21]. After 2-hour establishment of MCAO rat model that conformed to the method in a preceding study [22], the rats were performed with stereotactic injection of exosomes $(100 \mu \mathrm{g} / \mathrm{kg})$ and lentivirus $\left(5 \times 10^{7} \mathrm{TU} / \mathrm{mL}\right)$ into lateral ventricle [23]. After 72 hours, rats were euthanized and the brain tissue was collected.

\section{Neurological function score}

After 24 hours of animal sobriety, the neurological function was appraised by two observers through blind trial. The neurological deficit in MCAO rats was classified into five levels as follows: 0 score, no obvious neurological deficit symptoms; 1 score, when the rats were pulled up, the left forelimb could not be fully extended; 2 scores, when the tail was pull up, the left forelimb flexed; when rats were put on the ground to left, the crawling resistance was lower than the right; 3 scores, when the crawling condition was the same as 2 scores, the rats displayed involuntary left turn; 4 scores, coma, including death within 24 hours.

\section{Hematoxylin-eosin (HE) staining}

Samples were obtained for histologic examination after 24-hour reperfusion. The brains (5 in each group) were collected and the paraffin-embedded tissues were sliced into 6-mm coronal sections at $4^{\circ} \mathrm{C}$ after dehydration and hydration. The tissue sections were dewaxed with xylene, rehydrated with gradient 
ethanol and stained with hematoxylin and eosin. Then, the sections were observed under a light microscope (Olympus, Tokyo, Japan).

\section{Nissl staining}

Paraffin sections were dewaxed in xylene and hydrated with graded ethanol ( $95 \%, 85 \%$ and $75 \%)$. After rinsing with distilled water, the slices were stained with $0.5 \%$ crystal violet for 10 minutes and observed under the BX53 light microscope (Olympus).

\section{Transferase-mediated deoxyuridine triphosphate-biotin nick end labeling (TUNEL) staining}

The cell apoptosis in brain tissue was analyzed using TUNEL apoptosis detection kit (C1098, Beyotime Institute of Biotechnology, Shanghai, China). Paraffin sections were dewaxed and hydrated, and 20 $\mathrm{mg} / \mathrm{mL}$ DNase-free protease $\mathrm{K}$ was added dropwise. Then, the mixture was restored for 20 minutes and incubated with $3 \%$ hydrogen peroxide solution for 20 minutes. Then, $50 \mathrm{~mL}$ TUNEL working solution was added and restored for 60 minutes. The sample was added wit $0.3 \mathrm{~mL}$ labeled reaction termination solution and incubated for 10 minutes. Then, $50 \mathrm{~mL}$ streptavidin-horseradish peroxidase working solution was added with 15-minute incubation. Thereafter, $0.5 \mathrm{~mL}$ diaminobenzidine color solution was added for 30-minute incubation. After counter-staining of hematoxylin, ethanol gradient dehydration, xylene permeabilization and neutral resin sealing, the samples were observed under a light microscope. The cells with brown or brown-yellow nuclei and unstained cytoplasm were TUNEL-positive cells [24].

\section{Enzyme-linked immunosorbent assay (ELISA)}

Protein samples from the parietal cortex were collected after 24-hour reperfusion. The tumour necrosis factor alpha (TNF-a), interleukin-1beta (IL-1 $\beta$ ) and IL-6 levels were determined by ELISA kits (Nanjing KeyGen Biotech, Jiangsu, Chain) [25].

\section{Reverse transcription quantitative polymerase chain reaction (RT-qPCR)}

RNApure kit (BioTeKe corporation, Jiangsu, China). was used for extracting total RNA from brain tissue. To determine miR-150-5p or BTG2 mRNA expression, RT-qPCR was conducted by M-MIV reverse transcriptase (2641a, Takara Bio Inc., Otsu, Shiga, Japan) and SyBr ${ }^{\circledR}$ Green (BioTeke corporation). Relative gene expression was examined by $2^{-\triangle \Delta C t}$ method. The primers for miR-150-5p, BTG2 and glyceraldehyde-3-phosphate dehydrogenase (GAPDH) were shown in Table 1 [26]. 
Table 1

Primer sequences

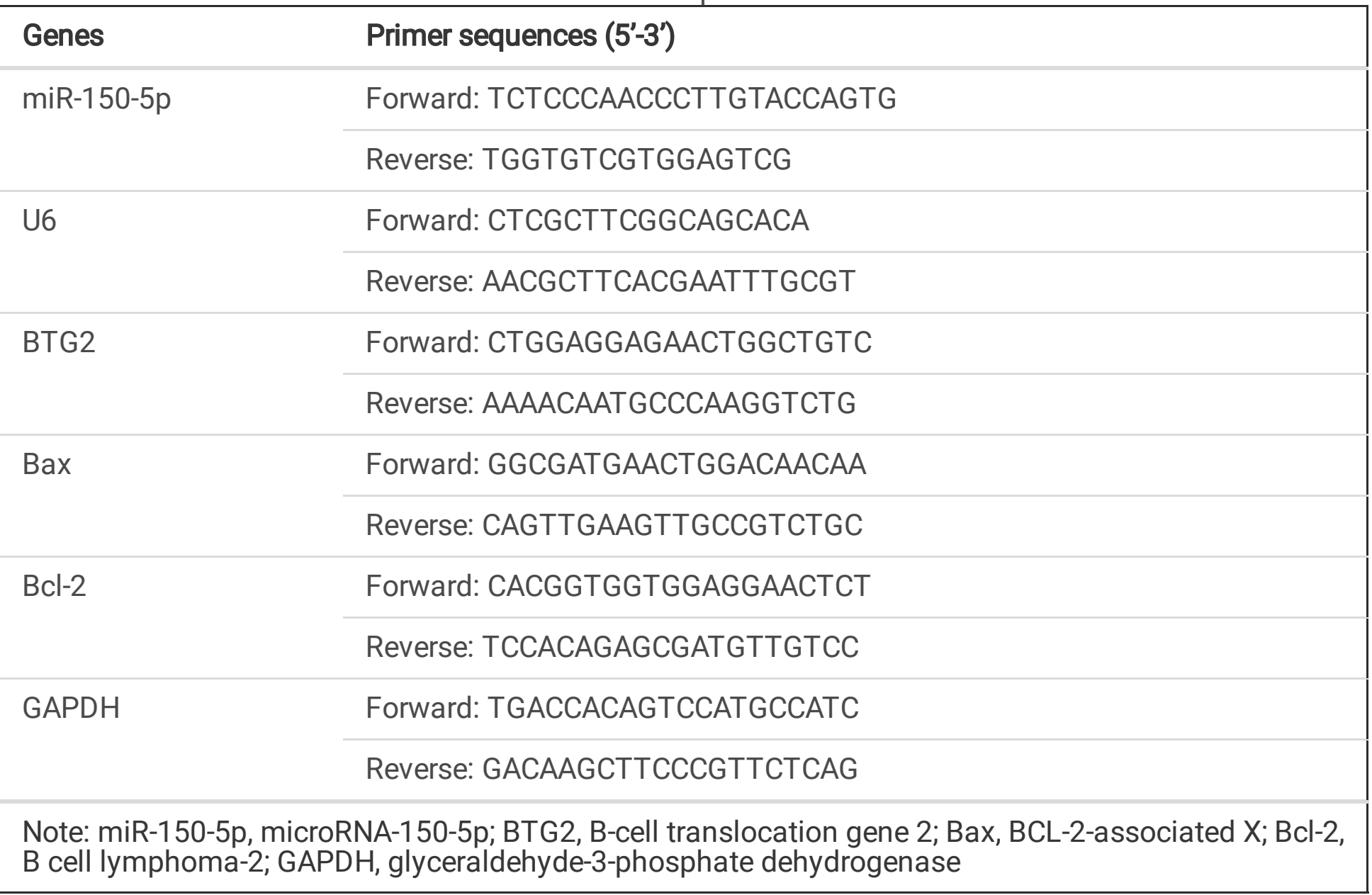

\section{Western blot assay}

Brain tissue was collected and lysed with $200 \mathrm{~mL}$ radio-immunoprecipitation assay cell lysis buffer (Santa Cruz Biotechnology, Santa Cruz, CA, USA, Dallas, Texas, USA). Solution was put into an EP tube and incubated on ice for 40 minutes. The EP tube was then centrifuged at $10000 \mathrm{rpm}$ for 20 minutes. Protein concentrations were examined through the bicinchoninic acid method. The protein and sample buffer were performed with sodium dodecyl sulphate polyacrylamide gel electrophoresis and transferred to polyvinylidene fluoride (PVDF) membrane. The PVDF membrane was sealed in a blocking buffer consisting of tris-buffered saline with Tween 20 (TBST) and 5\% skim milk. Then, the membrane was placed on a shaking table for 1 hour and incubated overnight with primary antibodies at $4^{\circ} \mathrm{C}$. GAPDH (1:5000, ab8245, Abcam Inc., Cambridge, MA, USA) and BTG2 (1:1000, ab197362, Abcam Inc.) were prepared for immunoblotting. Thereafter, the PVDF membrane was incubated overnight at $4^{\circ} \mathrm{C}$ in horseradish peroxidase conjugated goat-anti-rabbit and washed again with TBST for 10 minutes. Protein blots were quantified by software Quantity One (Bio-Rad Laboratories). The data was normalized to GAPDH [27].

\section{Dual luciferase reporter gene assay}


The targeting relationship between miR-150-5p and BTG2 was predicted through the bioinformatic website Starbase. Mutant (MUT) BTG2 was used to compare the fluorescence intensity with wild-type (WT) BTG2 that bound with miR-150-5p, validating that BTG2 was targeted by miR-150-5p. For the 3'untranslated region (UTR) assay, 293T cells with stably expressed miR-150-5p were cultured in 96-well plates and co-transfected with 50 ng BTG2-3'-UTR-WT or BTG2-3'UTR-MUT and control luciferase reporter gene (10 ng pRLCMV renilla luciferase reporter gene) by Lipofectamine 2000 (Thermo Fisher Scientific Inc). After 48 hours, cell lysates were collected. The fireflies and renilla luciferase activity was measured through the dual luciferase report analysis system (Promega, Madison, WI, USA) [20].

\section{Statistical Analysis}

The data were expressed as mean \pm standard deviation, and each experiment was repeated at least 3 times. The statistical analysis was conducted by GraphPad Prism 8.0 (GraphPad Software, Inc., CA, USA). The unpaired t-test was used for the comparison between two groups; one-way analysis of variance (ANOVA) was adopted for comparisons among multiple groups and followed by Tukey's post hoc test for pairwise comparison. $P<0.05$ was an indicator of statistical significance [26].

\section{Results}

\section{The identification of BMSCs and exosomes}

The BMSCs were mainly consisted of spindle-shaped cells with single and uniform shape. The BMSCs were arranged in a vortex-like and radial manner, and conformed to the typical growth pattern of BMSCs (Fig. 1A). The outcomes of flow cytometry showed that CD29, D54 and CD90 were positively expressed and CD45 was negatively expressed (Fig. 1B). The results of oil red $\mathrm{O}$ staining and alizarin red staining implied that lipid droplets were stained red. The enriched red droplets represented more fat formation, whereas the less droplets represented suppressed osteogenic effects. Oil-red 0 staining (Fig. 1C, D) reflected a significant increase in the number of lipid droplets from BMSCs; the osteogenic differentiation ratio of BMSCs was reduced as evidenced by alizarin-red staining.

Exosomes were extracted from the culture medium of BMSCs. The results of nanoparticle tracking analysis (NTA) particle size detection revealed (Fig. 1E) that the exosomes of BMSCs were mainly 80$120 \mathrm{~nm}$ in diameter. Exosome morphology was observed under the transmission electron microscope (Fig. 1F), which exhibited several saucer-shaped vesicles with a diameter of 80-120 nm. The surface markers of BMSCs were identified by Western blot assay (Fig. 1G). The results uncovered that the expression of CD63, CD9 and CD81 was positive, indicating that exosomes were successfully isolated.

The detection of RT-qPCR implied that miR-150-5p level in BMSCs transfected with miR-150-5p-mimic was significantly augmented (Fig. $1 \mathrm{H})$.

\section{Exosomes can effectively mitigate cerebral I/R injury}


It has been reported that exosomes exert protective effects on cerebral I/R injury [28], but the potential mechanism of BMSCs-exosomes in protection of cerebral I/R injury was unclear. In order to further explain the mechanism, the MCAO rat model was constructed by modified nylon suture method, and the neurological function of MCAO rats was scored by single blind trail. The results showed that (Fig. $2 A)$ the score of MCAO rats was saliently high, and the score of MCAO rats was low after exosomes injection. Subsequently, the pathological and histological lesions of the rat brain was examined through HE staining, Nissl staining and TUNEL staining. The outcome of HE staining (Fig. 2B) demonstrated that the pathological changes were obvious in MCAO rats, which displayed necrotic and degenerated neurons with disordered arrangement, concentrated neuron cytoplasm, condensed and unclear nucleus. However, in MOCA rats injected with exosomes, the neurons were less lost and damaged, only few neurons showed degeneration and necrosis. The results of Nissl staining implied that (Fig. 2C) the structure of neurons in MCAO rats was destroyed during I/R, the number of Nissl body decreased. In MCAO rats injected with exosomes, the quantity of Nissl body was augmented. The results of TUNEL staining mentioned that (Fig. 2D) there were more apoptotic cells in MCAO rats, while the apoptotic cells in MOCA rats injected with exosomes was obviously reduced. In addition, RT-qPCR was adopted to examine the apoptosisrelated genes level in brain tissue of rats. The results implied that (Fig. 2E) B cell lymphoma-2 (Bcl-2)associated X (Bax) mRNA was enriched and Bcl-2 mRNA level was depleted in MCAO rats; while Bax mRNA level was reduced and Bcl-2 mRNA level was elevated in rats injected with exosomes. The levels of inflammatory factors were assessed by ELISA (Fig. 2F), which indicated that the levels of TNF-a, IL-1 $\beta$ and IL- 6 were significantly enriched in the brain of the MCAO rats while reduced in the brain of the rats injected with exosomes.

The results validated that the MCAO rat model was successfully constructed, and the exosomes secreted by BMSCs could effectively protect the brain injury.

\section{Elevated miR-150-5p promotes BMSCs-exosomes- mediated protective effects on cerebral I/R injury}

It has been elucidated that miR-150 regulates the protection of myocardial ischemia injury [29], so we speculated that miR-150 might also be involved in exosomes-mediated protection of cerebral I/R injury. We examined the expression of miR-150-5p first (Fig. 3A), and it was found that miR-150-5p was lowexpressed in MCAO rats and its expression was elevated after injection with exosomes by RT-qPCR. Next, exosomes from BMSCs that transfected with miR-150-5p-mimic were injected into the MCAO rats, the outcomes implied that miR-150-5p was augmented in rats injected with Exo + miR-150-5p-mimic. In response to the enriched miR-150-5p, the neurological function score was lowered (Fig. 3B); the cell arrangement tended to be regular with decreased necrotic neurons (Fig. 3C); the quantity of Nissl body was increased (Fig. 3D); the apoptotic cells decreased (Fig. 3E); the Bax mRNA level was decreased and Bcl-2 mRNA level was augmented (Fig. 3F) and the levels of TNF-a, IL-1 $\beta$ and IL-6 were reduced (Fig. 3G).

The results showed that miR-150-5p was silenced in rats with cerebral I/R injury. The exosomes injection elevated the miR-150-5p expression. The induction of miR-150-5p could further facilitate the therapeutic 
efficiency of BMSCs-exosomes for cerebral I/R injury.

\section{BTG2 is a target gene of miR-150-5p}

Next, we studied the downstream target genes of miR-150-5p. A previous finding has clarified that BTG2 exerts effects on ischemic stroke [30]. The Starbase predicted that there was a targeting relationship between miR-150-5p and BTG2 and the binding site was predicated (Fig. 4A). Subsequently, the targeting relationship between miR-150-5p and BTG2 was validated through the dual luciferase reporting assay (Fig. 4B), which uncovered that the luciferase activity of 293T cells was depleted after the co-transfection of BTG2-WT and miR-150-5p-mimic.

To further verify the targeting relationship between miR-150-5p and BTG2, we detected BTG2 expression in rats by RT-qPCR and Western blot assay. The results implied that BTG2 was low-expressed in rats injected with exosomes from BMSCs that transfected with miR-150-5p-mimic (Fig. 4C).

The results showed that BTG2 was a downstream target gene of miR-150-5p, and the elevation of miR150-5p inhibited BTG2 expression.

\section{Inhibited BTG2 enhances the BMSCs-exosomes-mediated protective effects on cerebral I/R injury}

The BTG2 level in rat brain tissue was assessed by RT-qPCR and Western blot assay. The results demonstrated that (Fig. 5A) BTG2 was elevated in MCAO rats, while BTG2 was decreased after the injection of exosomes, meanwhile, in rats injected with Exo + sh-BTG2, BTG2 was drastically reduced. As a result of decreased BTG2, the rats displayed low neurological function score, fewer necrotic neurons, more Nissl bodies, less apoptotic cells, reduced Bax mRNA level, enhanced Bcl-2 mRNA level and ablated TNF- $\alpha$, IL-1 $\beta$ and IL-6 level (Fig. 5B-G).

The outcome reflected that silenced BTG2 could strengthen the therapeutic effect of exosomes. BTG2 was involved in the protection of BMSCs-exosomes-mediated protection on cerebral I/R injury.

\section{Elevated BTG2 inhibits the therapeutic effect of exosomal miR-150-5p from BMSCs on cerebral I/R injury}

To probe whether exosomal miR-150-5p from BMSCs protects against cerebral I/R injury by regulating the expression of BTG2, exosomes from BMSCs that transfected with miR-150-5p-mimic as well as the oeBTG2 lentivirus were co-transfected into MCAO rats. The result of RT-qPCR and Western blot assay validated that BTG2 was increased in rats injected with Exo + miR-150-5p-mimic + oe-BTG2 (Fig. 6A). The rats with enriched BTG2 exhibited high neurological function score, numerous necrotic and degenerated neurons, disordered arrangement, concentrated neuron cytoplasm, condensed and unclear nuclei, destroyed neuronal cells, less Nissl bodies, more apoptotic cells, elevated Bax mRNA level, reduced Bcl-2 mRNA level and enriched levels of TNF-a, IL-1 $\beta$ and IL-6 (Fig. 6B-G). These outcomes validated that augmented BTG2 reversed the effects of enhanced miR-150-5p on cerebral I/R injury. 
It was uncovered that the enrichment of BTG2 inhibited the therapeutic influence of exosomal miR-150-5p from BMSCs on cerebral I/R injury.

\section{Discussion}

Cerebral ischemia is one of the most malignant complications in the perioperative period with severe clinical sequelae [31]. In this study, we focused on the regulatory mechanism of exosomal miR-150-5p from BMSCs on the progression of cerebral I/R injury through mediating BTG2. Collectively, it was demonstrated that exosomal miR-150-5p from BMSCs facilitated the protective effects on cerebral I/R injury by suppressing BTG2.

A previous study have demonstrated that the median loglow-expressed miR-150-5p in ischemic stroke is accountable for unfavorable outcome and high mortality of patients [32] Additionally, it has been uncovered that miR-150-5p is depleted in patients with advanced heart failure, and the reduction of miR150-5p induces severe clinical exacerbation, leading to death from progressive pump failure or even requiring hospitalization [33]. Furthermore, the overexpression of miR-150-5p effectively enhances the Bax level as well as the cell viability and invasion of mouse embryonic cardiomyocytes H9c2 cells [34]. Concretely, miR-150-5p also exhibits low level in rats with myocardial I/R injury, and the extracellular vesicles derived from miR-150-5p effectively suppress the cardiomyocyte apoptosis and myocardial remodeling, thus to favor the treatment of I/R [15]. In addition, it has been verified the exosomes from BMSCs can attenuate renal I/R injury [10]. Identically, another literature has also implied that exosomes extracted from BMSCs activate cell viability in oxygen-glucose deprivation/reoxygenation and constrain the pyroptosis, thereby mitigating the cerebral I/R injury [7]. Similarly, Wu et al. have unearthed that miR150-5p from exosomes of BMSCs can protect cardiac function, mitigate pathological changes of myocardium and reduce apoptosis rate of cardiomyocytes in myocardial infarction mice [35]. However, the literatures about effects of the exosomal miR-150-5p from BMSCs on cerebral I/R injury are inadequate, yet these preceding findings still offered valuable references and basement for our study. Anchored in these evidences, this study validated that exosomes could effectively attenuate the cerebral I/R injury in MOCA rats. Furthermore, miR-150-5p is depleted in MCAO rats, and the augmented miR-150$5 p$ enhanced the protective impact of exosomes derived from BMSCs on cerebral I/R injury.

Furthermore, the binding relationship between miR-150-5p and BTG2 was predicated and clarified by the bioinformatic website and the dual luciferase reporter gene assay. It has been reflected that BTG2 is enriched after the ischemic stress, and the depletion of BTG2 can increase the abundances of astrocytes and the proliferation of glial cells during the cerebral hypoperfusion [16]. Similarly, Gubern et al. have raveled that BTG2 level exhibits sixfold increase after ischemia in MCAO rats [36]. Clinically, Wu et al. have elucidated that BTG2 is enriched in the renal medullary tissue samples of hypertensive patients, whereas the elevation of BTG2 leads to functional abnormalities and induces the occurrence of hypertensive disorders [37]. Furthermore, it has reported that the loss of BTG2 effectively facilitates the proliferation of cardiomyocyte in mouse with myocardial infarction [38]. The silence of BTG2 can mitigate neuronal apoptosis and relieve oxidative stress in brain tissues of rats with postoperative 
cognitive dysfunction [39]. Other studies also have implied that BTG2 is enriched in rats after the retinal ischemia and reperfusion and after focal ischemia [36, 40]. However, there existed limited studies about the role of BTG2 on BMSCs-exosomes-mediated protective effects on cerebral I/R injury. Based on previous findings, the study further explored the function of BTG2 on cerebral I/R injury. It was demonstrated that BTG2 was enriched in MCAO rats, and silenced BTG2 could strengthen the protective effects of BMSCs-exosomes on cerebral I/R injury.

Collectively, this study reveals that miR-150-5p is decreased while BTG2 is enriched in MCAO rats, and the exosomal miR-150-5p from BMSCs can exert protective effect on cerebral I/R injury via reducing BTG2. The current discovery makes a contribution for exploring novel therapy strategies for cerebral I/R injury by highlighting the importance of exosomal miR-150-5p from BMSCs and BTG2. However, the study samples still need further enrichment to get more precise data for a convincing conclusion.

\section{Declarations}

\section{Conflict of interest}

The authors declare that they have no conflicts of interest.

\section{Acknowledgement}

We thank the associate editor and the reviewers for their useful feedback that improved this paper.

\section{References}

1. Shin, T.H., et al., Metabolome Changes in Cerebral Ischemia. Cells, 2020. 9(7).

2. Yang, Z., et al., Protective effects of mangiferin on cerebral ischemia-reperfusion injury and its mechanisms. Eur J Pharmacol, 2016. 771: p. 145-51.

3. Catanese, L., J. Tarsia, and M. Fisher, Acute Ischemic Stroke Therapy Overview. Circ Res, 2017. 120(3): p. 541-558.

4. Eltzschig, H.K. and T. Eckle, Ischemia and reperfusion-from mechanism to translation. Nat Med, 2011. 17(11): p. 1391-401.

5. $\quad \mathrm{Li}, \mathrm{H}$., et al., Isolation and characterization of primary bone marrow mesenchymal stromal cells. Ann N Y Acad Sci, 2016. 1370(1): p. 109-18.

6. Wang, J., et al., Combined bone marrow stromal cells and oxiracetam treatments ameliorates acute cerebral ischemia/reperfusion injury through TRPC6. Acta Biochim Biophys Sin (Shanghai), 2019. 51(8): p. 767-777. 
7. Zeng, Q., et al., Exosomes Secreted From Bone Marrow Mesenchymal Stem Cells Attenuate Oxygen-Glucose Deprivation/Reoxygenation-Induced Pyroptosis in PC12 Cells by Promoting AMPKDependent Autophagic Flux. Front Cell Neurosci, 2020. 14: p. 182.

8. Pegtel, D.M. and S.J. Gould, Exosomes. Annu Rev Biochem, 2019. 88: p. 487-514.

9. $\mathrm{Yu}, \mathrm{H}$., et al., Hypoxic Preconditioning Enhances the Efficacy of Mesenchymal Stem Cells-Derived Conditioned Medium in Switching Microglia toward Anti-inflammatory Polarization in Ischemia/Reperfusion. Cell Mol Neurobiol, 2021. 41(3): p. 505-524.

10. Wang, C., et al., BMSCs protect against renal ischemia-reperfusion injury by secreting exosomes loaded with miR-199a-5p that target BIP to inhibit endoplasmic reticulum stress at the very early reperfusion stages. FASEB J, 2019. 33(4): p. 5440-5456.

11. Zhu, G., et al., Exosomes from human-bone-marrow-derived mesenchymal stem cells protect against renal ischemia/reperfusion injury via transferring miR-199a-3p. J Cell Physiol, 2019. 234(12): p. 23736-23749.

12. Chen, Q., et al., Bone marrow mesenchymal stem cell-secreted exosomes carrying microRNA-125b protect against myocardial ischemia reperfusion injury via targeting SIRT7. Mol Cell Biochem, 2020. 465(1-2): p. 103-114.

13. Saliminejad, K., et al., An overview of microRNAs: Biology, functions, therapeutics, and analysis methods. J Cell Physiol, 2019. 234(5): p. 5451-5465.

14. He, Q.W., et al., MiR-150 Regulates Poststroke Cerebral Angiogenesis via Vascular Endothelial Growth Factor in Rats. CNS Neurosci Ther, 2016. 22(6): p. 507-17.

15. Ou, $\mathrm{H}$., et al., Extracellular vesicles derived from microRNA-150-5p-overexpressing mesenchymal stem cells protect rat hearts against ischemia/reperfusion. Aging (Albany NY), 2020. 12(13): p. 1266912683.

16. Suzuki, K., et al., Deletion of B-cell translocation gene 2 (BTG2) alters the responses of glial cells in white matter to chronic cerebral hypoperfusion. J Neuroinflammation, 2021. 18(1): p. 86.

17. Ohtomo, R., et al., Cilostazol alleviates white matter degeneration caused by chronic cerebral hypoperfusion in mice: Implication of its mechanism from gene expression analysis. Neurosci Lett, 2018. 662: p. 247-252.

18. Fang, S., Y. Li, and P. Chen, Osteogenic effect of bone marrow mesenchymal stem cell-derived exosomes on steroid-induced osteonecrosis of the femoral head. Drug Des Devel Ther, 2019. 13: p. 45-55.

19. Yan, W., et al., Cancer-cell-secreted exosomal miR-105 promotes tumour growth through the MYCdependent metabolic reprogramming of stromal cells. Nat Cell Biol, 2018. 20(5): p. 597-609. 
20. Yu, Y., et al., Expression and regulation of miR-449a and AREG in cerebral ischemic injury. Metab Brain Dis, 2019. 34(3): p. 821-832.

21. Zhang, D., et al., Microglia exosomal miRNA-137 attenuates ischemic brain injury through targeting Notch1. Aging (Albany NY), 2021. 13(3): p. 4079-4095.

22. Zhang, G., et al., Wnt/beta-catenin signaling pathway contributes to isoflurane postconditioning against cerebral ischemia-reperfusion injury and is possibly related to the transforming growth factorbeta1/Smad3 signaling pathway. Biomed Pharmacother, 2019. 110: p. 420-430.

23. Huang, X., et al., Exosomes derived from PEDF modified adipose-derived mesenchymal stem cells ameliorate cerebral ischemia-reperfusion injury by regulation of autophagy and apoptosis. Exp Cell Res, 2018. 371(1): p. 269-277.

24. Zhang, M., et al., miR-137 alleviates focal cerebral ischemic injury in rats by regulating JAK1/STAT1 signaling pathway. Hum Exp Toxicol, 2020. 39(6): p. 816-827.

25. Li, M., et al., MiRNA-199a-5p Protects Against Cerebral Ischemic Injury by Down-Regulating DDR1 in Rats. World Neurosurg, 2019. 131: p. e486-e494.

26. Zhu, L., et al., miR1835p attenuates cerebral ischemia injury by negatively regulating PTEN. Mol Med Rep, 2020. 22(5): p. 3944-3954.

27. Wang, S.L., et al., Dexmedetomidine preconditioning plays a neuroprotective role and suppresses TLR4/NF-kappaB pathways model of cerebral ischemia reperfusion. Biomed Pharmacother, 2017. 93: $p$. 1337-1342.

28. Jiang, Y., et al., Plasma exosomes protect against cerebral ischemia/reperfusion injury via exosomal HSP70 mediated suppression of ROS. Life Sci, 2020. 256: p. 117987.

29. Tang, Y., et al., MicroRNA-150 protects the mouse heart from ischaemic injury by regulating cell death. Cardiovasc Res, 2015. 106(3): p. 387-97.

30. Slevin, M., et al., B-cell translocation gene 2 is over-expressed in peri-infarct neurons after ischaemic stroke. Pathobiology, 2009. 76(3): p. 129-35.

31. Zhou, Z.B., et al., Cerebral ischemia during surgery: an overview. J Biomed Res, 2016. 30(2): p. 8387.

32. Scherrer, N., et al., MicroRNA 150-5p Improves Risk Classification for Mortality within 90 Days after Acute Ischemic Stroke. J Stroke, 2017. 19(3): p. 323-332.

33. Scrutinio, D., et al., Circulating microRNA-150-5p as a novel biomarker for advanced heart failure: $A$ genome-wide prospective study. J Heart Lung Transplant, 2017. 36(6): p. 616-624. 
34. Zhou, J., et al., LnCRNA XIST inhibits hypoxia-induced cardiomyocyte apoptosis via mediating miR-150-5p/Bax in acute myocardial infarction. Eur Rev Med Pharmacol Sci, 2020. 24(3): p. 1357-1366.

35. Wu, Z., et al., BMSCs-derived exosomal microRNA-150-5p attenuates myocardial infarction in mice. Int Immunopharmacol, 2021. 93: p. 107389.

36. Gubern, C., et al., miRNA expression is modulated over time after focal ischaemia: up-regulation of miR-347 promotes neuronal apoptosis. FEBS J, 2013. 280(23): p. 6233-46.

37. Wu, Y.B., et al., Analysis of FOS, BTG2, and NR4A in the function of renal medullary hypertension. Genet Mol Res, 2013. 12(3): p. 3735-41.

38. Xiang, F.L., M. Guo, and K.E. Yutzey, Overexpression of Tbx20 in Adult Cardiomyocytes Promotes Proliferation and Improves Cardiac Function After Myocardial Infarction. Circulation, 2016. 133(11): p. 1081-92.

39. Mao, L., et al., Elevation of miR-146a Inhibits BTG2/BAX Expression to Ameliorate Postoperative Cognitive Dysfunction Following Probiotics (VSL \#3) Treatment. Mol Neurobiol, 2021.

40. Prasad, S.S., et al., Retinal gene expression after central retinal artery ligation: effects of ischemia and reperfusion. Invest Ophthalmol Vis Sci, 2010. 51(12): p. 6207-19.

\section{Figures}


A

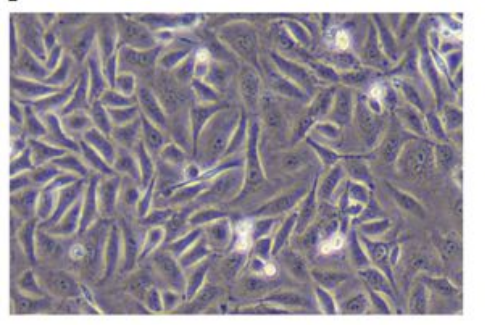

C Control

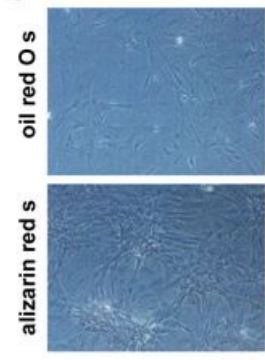

E
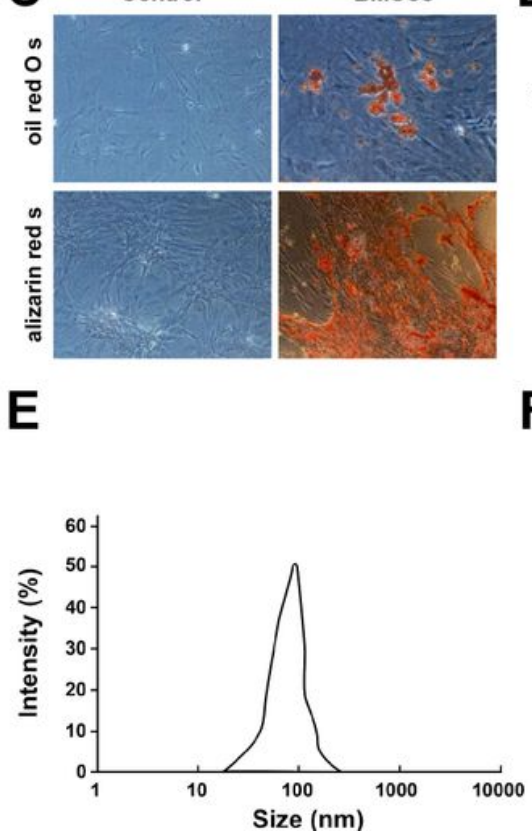

B
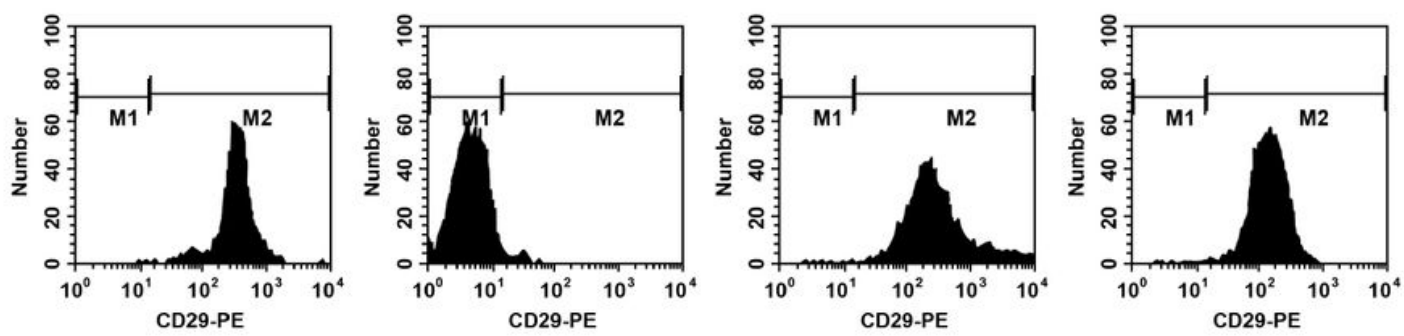

D

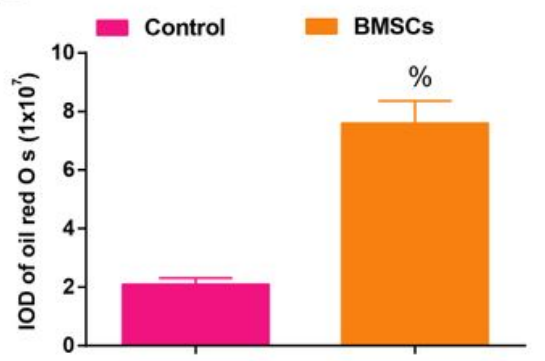

F

G

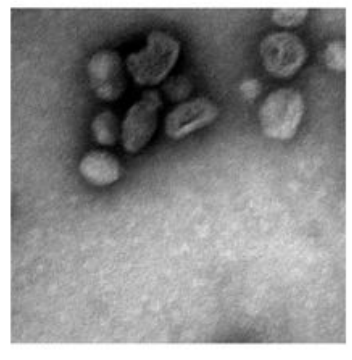

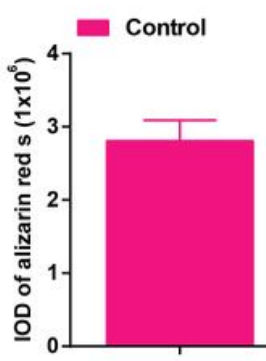

BMSCs

$\%$

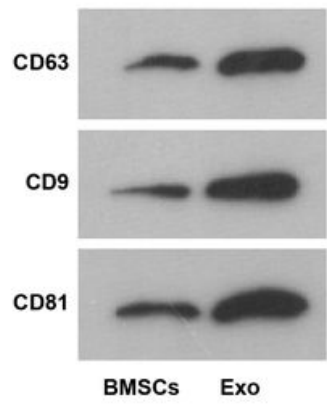

H

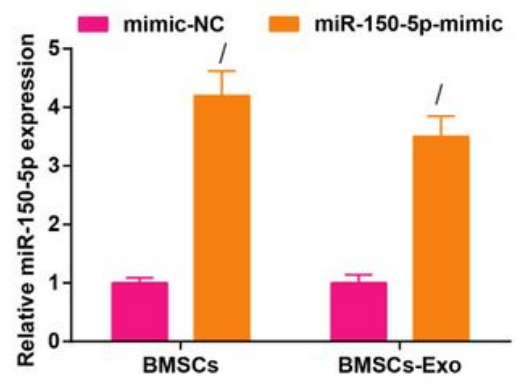

Figure 1

The identification of BMSCs and exosomes. A, observation of morphology of BMSCs (scale bar $=100$ $\mu \mathrm{m}) ; B$, identification of surface markers of BMSCs by flow cytometry; $C / D$, integrated OD of lipid droplets and osteogenic effects were examined by oil red $\mathrm{O}$ and alizarin red staining and calculated by Image-pro plus; $E$, the exosome particle size was measured by NTA; F, the exosome morphology was observed by transmission electron microscope (scale bar $=100 \mathrm{~nm}$ ); $\mathrm{G}$, the exosome surface markers were detected by Western blot assay; $\mathrm{H}$, miR-150-5p expression in BMSCs and exosomes was examined by RT-qPCR; $\mathrm{n}=$ $3 ; \% \mathrm{P}<0.05$ vs. the control group; / $\mathrm{P}<0.05$ vs. the mimic-NC group; each experiment was repeated at least 3 times; the data were expressed as mean \pm standard deviation; the unpaired t-test was used for the comparison between two groups. 


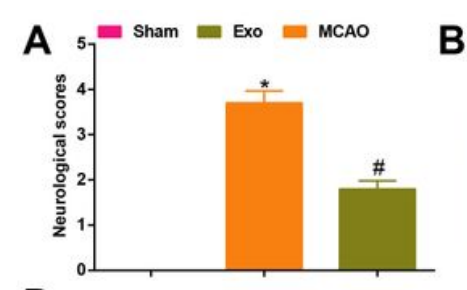

B
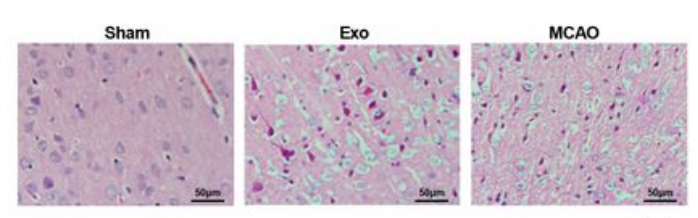

C

D
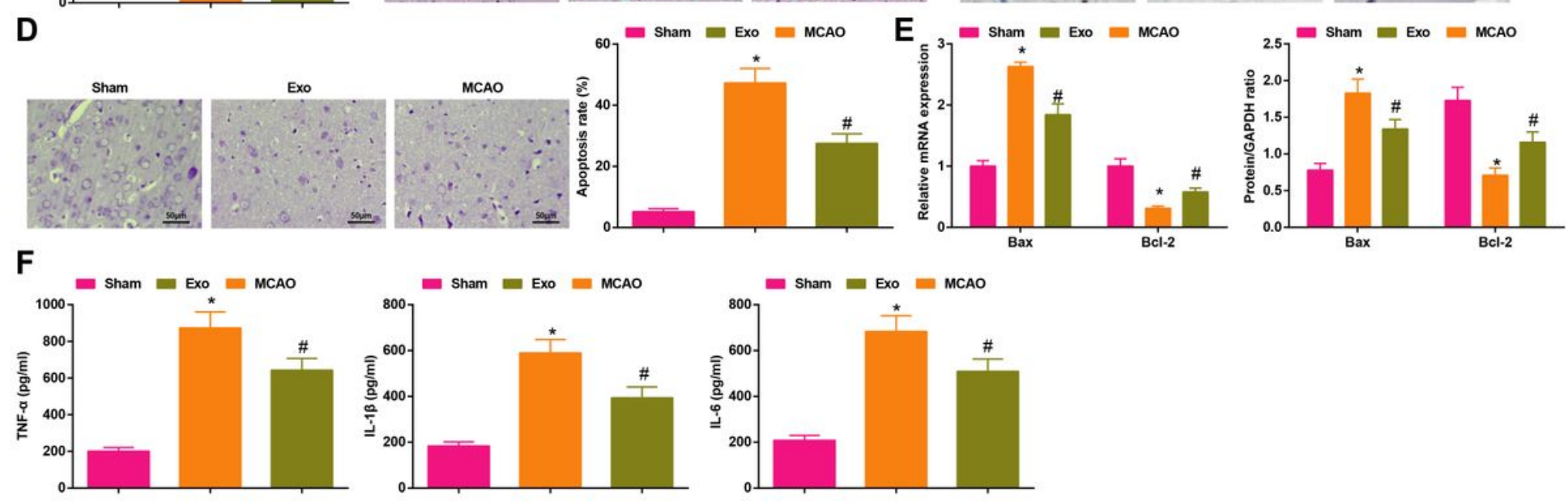

Figure 2

Exosomes can effectively mitigate cerebral I/R injury. A, the neurological function score of MCAO rats after injection with exosomes; $\mathrm{B}$, pathological tissue damage in rat brain after injection with exosomes was detected by HE staining (scale bar $=25 \mu \mathrm{m}$ ); C, the pathological damage in rat brain (scale bar $=50$ $\mu \mathrm{m})$ after injection with exosomes was examined by Nissl staining; D, cell apoptosis in rat brain after injection with exosomes was assessed by TUNEL staining (scale bar $=50 \mu \mathrm{m}$ ); E, Bax and Bcl-2 mRNA levels in rat brain after injection with exosomes were examined by RT-qPCR; $F$, the levels of inflammatory factors (TNF- $a, I L-1 \beta, I L-6)$ in rat brain was after injection with exosomes were detected by ELISA; A-F, $n=$ 6 ; * $\mathrm{P}<0.05$ vs. the Sham group; \# $\mathrm{P}<0.05$ vs. the MCAO group; the data were expressed as mean \pm standard deviation, the unpaired t-test was used for the comparison between two groups; one-way ANOVA was used for comparisons among multiple groups and Tukey's post hoc test was used for pairwise comparisons after one-way ANOVA. 

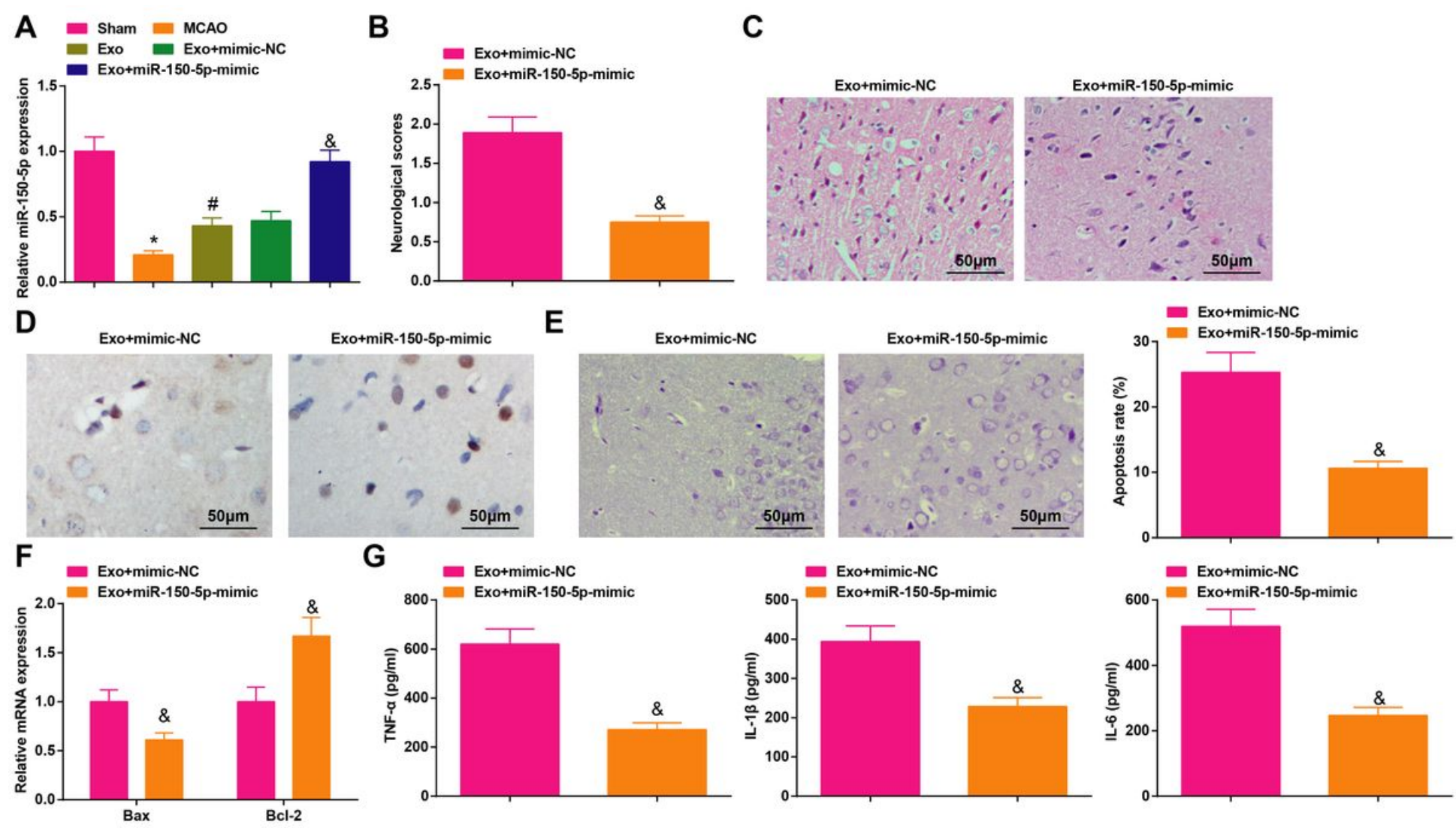

\section{Figure 3}

Elevated miR-150-5p promotes BMSCs-exosomes-mediated protective effects on cerebral I/R injury. A, miR-150-5p level in rat brain tissue was detected by RT-qPCR; B, the neurological function score in MCAO rats after injection with Exo + miR-150-5p-mimic; $C$, the pathological damage in rat brain (scale bar $=25$ $\mu \mathrm{m})$ after injection with Exo + miR-150-5p-mimic was examined by HE staining; $D$, the pathological damage in rat brain (scale bar $=50 \mu \mathrm{m}$ ) after injection with Exo + miR-150-5p-mimic was detected by Nissl staining; E, cell apoptosis in rat brain tissue (scale bar $=50 \mu \mathrm{m}$ ) after injection with Exo + miR-150$5 p$-mimic was assessed by TUNEL staining; $F$, the levels of Bax and Bcl-2 mRNA in rat brain tissue after injection with Exo + miR-150-5p-mimic were examined by RT-qPCR; $G$, the levels of inflammatory factors (TNF-a, IL-1 $\beta$, IL-6) in rat brain after injection with Exo + miR-150-5p-mimic were detected by ELISA; A-G, $n$ $=6$; the experiment was repeated at least three times; ${ }^{P}<0.05 \mathrm{vs}$. the Sham group; $\# \mathrm{P}<0.05 \mathrm{vs}$. the MCAO group; \& $P<0.05$ vs. the Exo + mimic-NC group. the data were expressed as mean \pm standard deviation, the unpaired t-test was used for the comparison between two groups. 
A

Binding Site of hsa-miR-150-5p on BTG2:

\begin{tabular}{|c|c|c|c|}
\hline BindingSite & $\uparrow$ & Class $\quad t \downarrow$ & Alignment \\
\hline chr1:203277775-203277780[+] & $\uparrow$ & $7 \mathrm{mer}-\mathrm{A} 11$ & $\begin{array}{c}\text { Target: } 5^{\prime} \text { uaunccuuggcugaaUGGGAGa } 3^{\prime} \\
1111111 \\
\text { miRNA : } 3^{\prime} \text { gugaccauguncccaACCCUCa } 5^{\prime}\end{array}$ \\
\hline
\end{tabular}

\section{C}

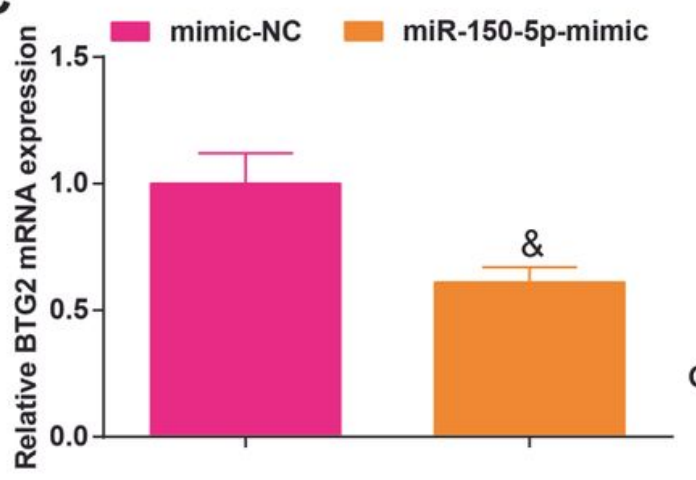

B

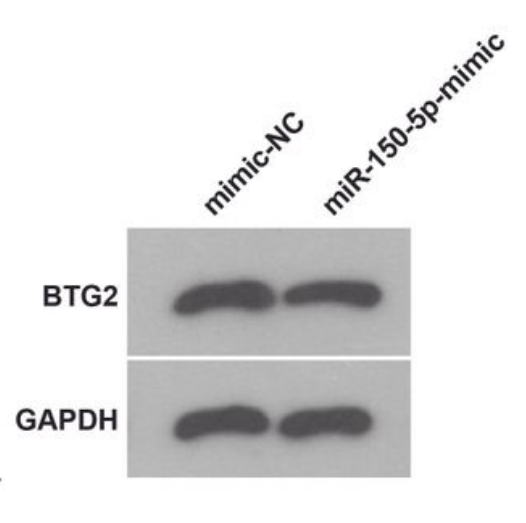

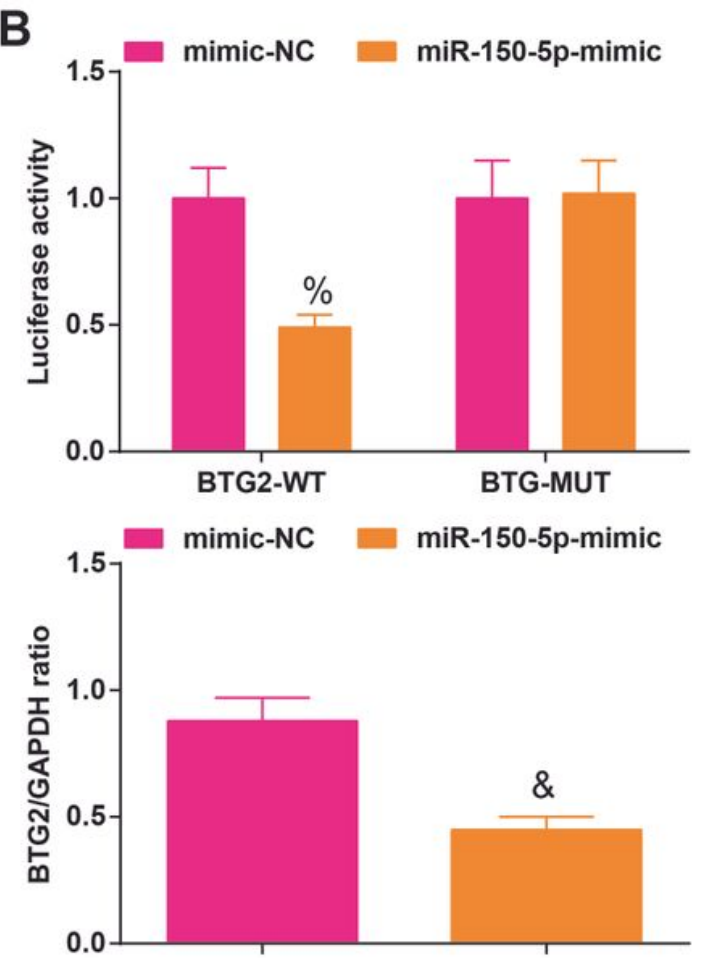

\section{Figure 4}

BTG2 is a target gene of miR-150-5p. A, the targeting relationship between miR-150-5p and BTG2 was predicted by Starbase; B, the targeting relationship between miR-150-5p and BTG 2 was validated by the dual luciferase reporter gene assay; C, BTG2 expression in rat brain tissue after injection with Exo + miR150-5p-mimic was detected by RT-qPCR and Western blot assay. A-B, N = 3; C, n =6; \% P< 0.05 vs. the mimic NC group; \& $\mathrm{P}<0.05$ vs. the Exo + mimic-NC group; the experiment was repeated at least three times; the data were expressed as mean \pm standard deviation, the unpaired $t$-test was used for the comparison between two groups. 

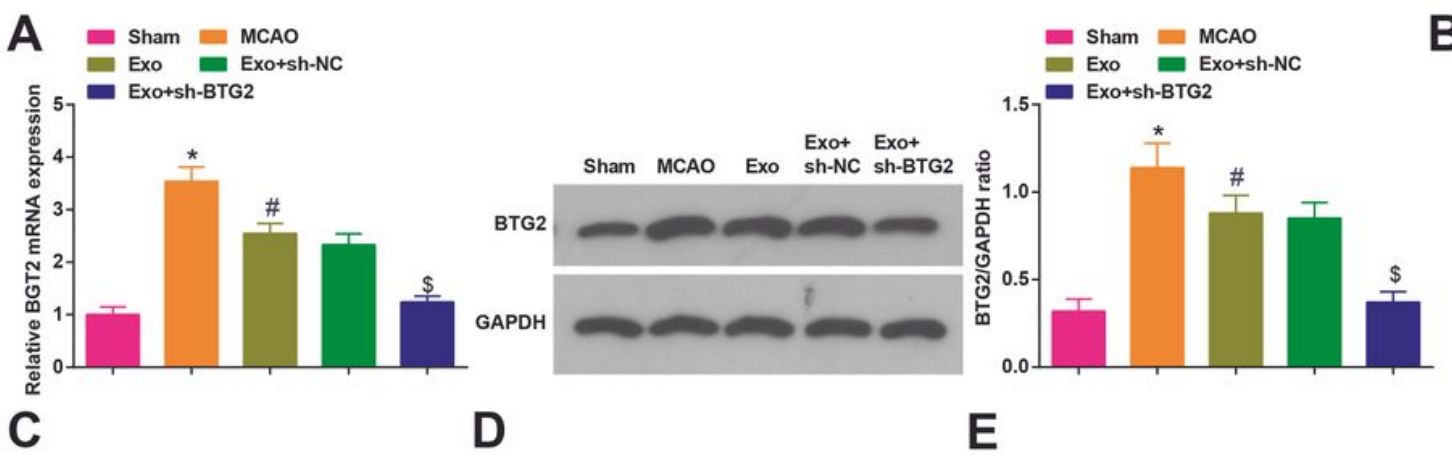

B

E
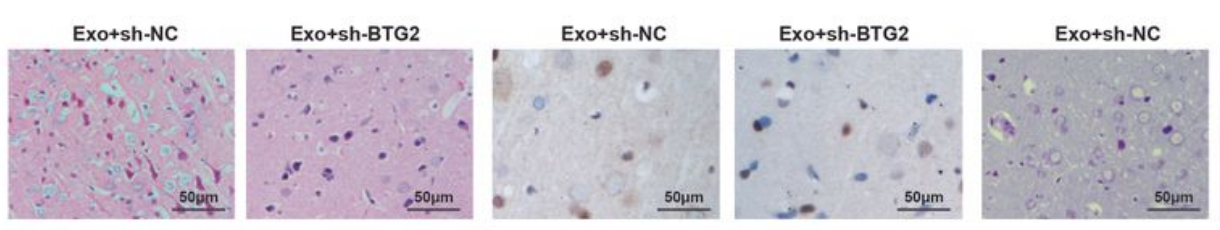

Exo+sh-BTG2
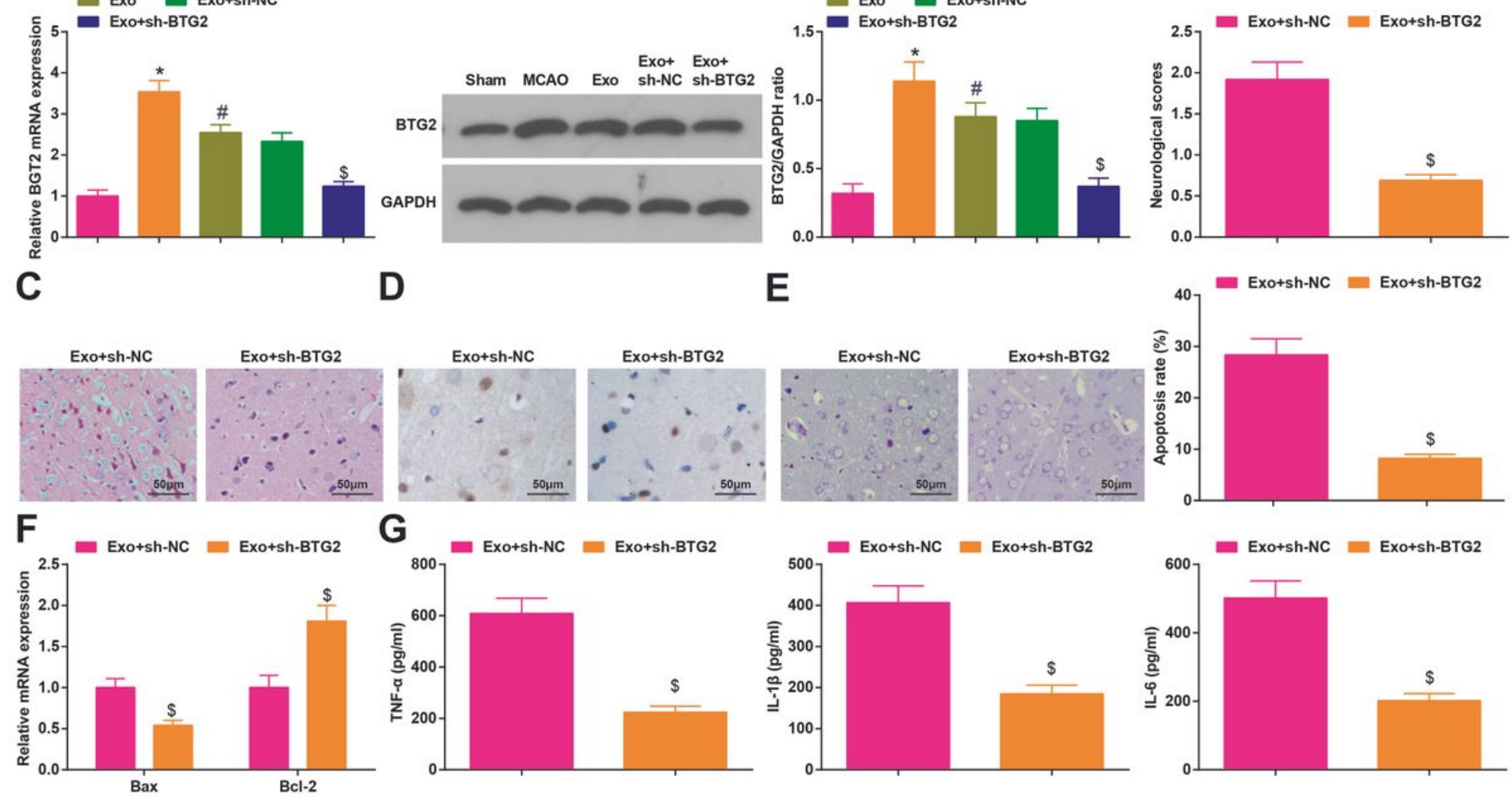

Figure 5

Inhibited BTG2 enhances the BMSCs-exosomes-mediated protective effects on cerebral I/R injury. A, BTG2 level in rat brain tissue was detected by RT-qPCR and Western blot assay; B, the neurological function score in MCAO rats after injection with Exo + sh-BTG2; $C$, the pathological damage in rat brain (scale bar $=25 \mu \mathrm{m}$ ) after injection with Exo + sh-BTG2 was examined by HE staining; D, the pathological damage in rat brain (scale bar $=50 \mu \mathrm{m}$ ) after injection with Exo + sh-BTG2 was detected by NissI staining; $E$, cell apoptosis in rat brain tissue (scale bar $=50 \mu \mathrm{m}$ ) after injection with Exo + sh-BTG2 was assessed by TUNEL staining; $F$, the levels of Bax and Bcl-2 mRNA in rat brain tissue after injection with Exo + sh-BTG2 were examined by RT-qPCR; $G$, the levels of inflammatory factors (TNF-a, IL-1 $\beta$, IL-6) in rat brain was after injection with Exo + sh-BTG2 were detected by ELISA; $A-G, n=6$; * $P<0.05$ vs. the sham group; \# P $<0.05$ vs. the MCAO group; $\$ \mathrm{P}<0.05$ vs. the Exo + sh-NC group; the experiment was repeated at least three times; the data were expressed as mean \pm standard deviation, the unpaired t-test was used for the comparison between two groups. 
A

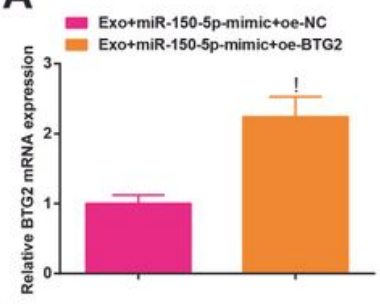

C

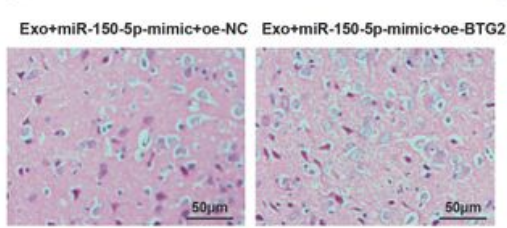

$\mathbf{F}=$ Exo+miR-150-5p-mimictoe-NC

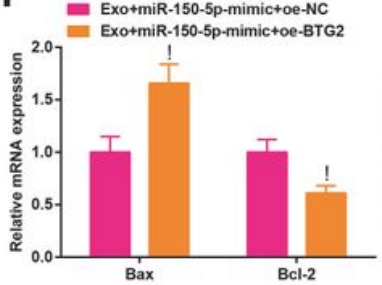

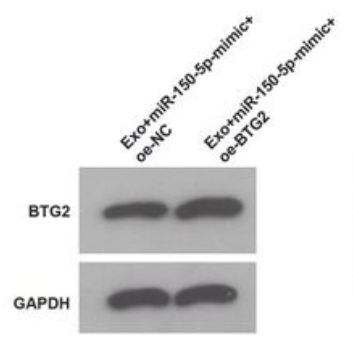

D

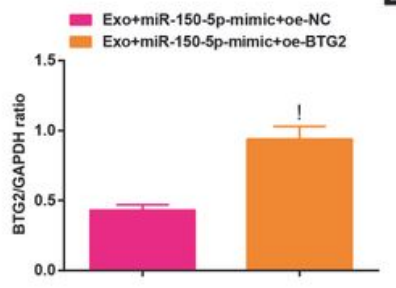

$E$
B

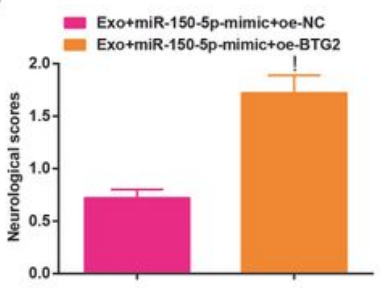

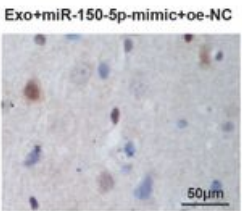
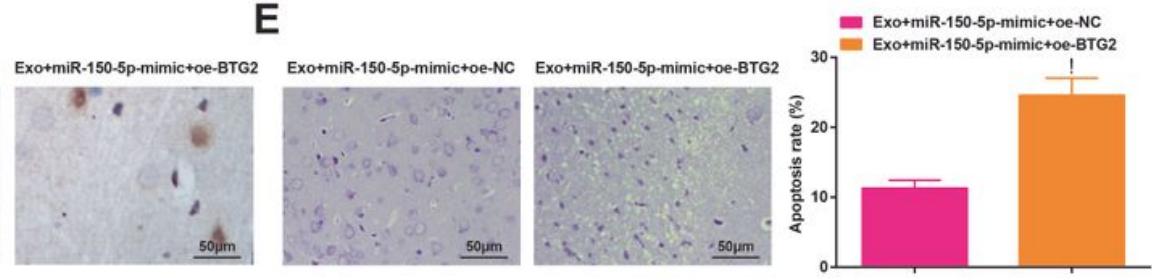

G
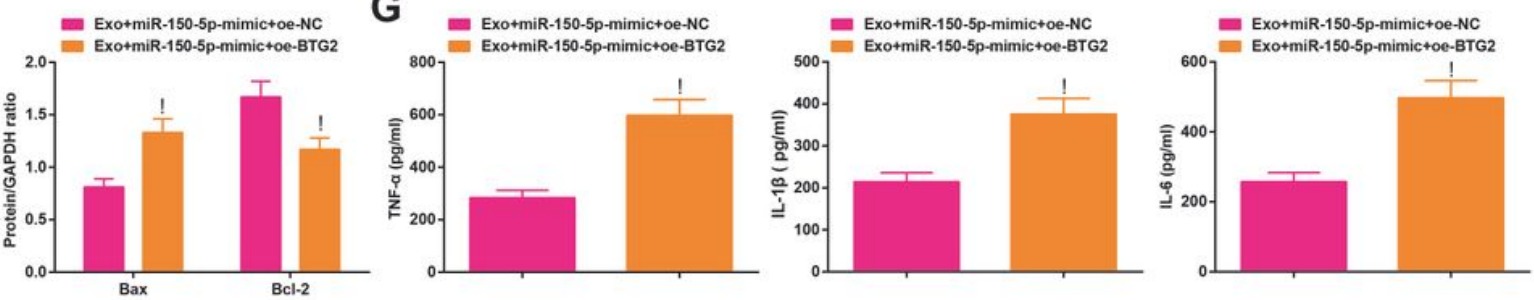

Figure 6

Elevated BTG2 inhibits the therapeutic effect of exosomal miR-150-5p from BMSCs on cerebral I/R injury. A, BTG2 level in rat brain tissue after injection with Exo + miR-150-5p-mimic + oe-BTG2 was detected by RT-qPCR and Western blot assay; $B$, the neurological function score in MCAO rats after injection with Exo + miR-150-5p-mimic + oe-BTG2; $C$, the pathological damage in rat brain (scale bar $=25 \mu \mathrm{m}$ ) after injection with Exo + miR-150-5p-mimic + oe-BTG2 was examined by HE staining; $D$, the pathological damage in rat brain (scale bar $=50 \mu \mathrm{m}$ ) after injection with Exo + miR-150-5p-mimic + oe-BTG2 was detected by Nissl staining; $\mathrm{E}$, cell apoptosis in rat brain tissue (scale bar $=50 \mu \mathrm{m}$ ) after injection with Exo + miR-150-5pmimic + oe-BTG2 was assessed by TUNEL staining; F, the levels of Bax and Bcl-2 mRNA in rat brain tissue after injection with Exo + miR-150-5p-mimic + oe-BTG2 were examined by RT-qPCR; G, the levels of inflammatory factors (TNF- $a$, IL-1 $\beta$, IL-6) in rat brain was after injection with Exo + miR-150-5p-mimic + oe-BTG2 were detected by ELISA; A-G, $n=6 ;$ ! P $<0.05$ vs. the Exo + miR-150-5p + mimic + oe-NC group; the experiment was repeated at least three times; the data were expressed as mean \pm standard deviation, the unpaired t-test was used for the comparison between two groups. 